\title{
Primer registro del género Interocoris (Heteroptera: Naucoridae) en Centroamérica y observaciones sobre Interocoris mexicanus
}

\author{
Federico Herrera ${ }^{1,2}$, Darha Solano-Ulate ${ }^{3} \&$ Monika Springer $^{3,4}$ \\ 1. Escuela de Ciencias Biológicas, Universidad Nacional, Heredia, Costa Rica; federico.herrera.madrigal@una.cr \\ 2. Escuela de Ciencias Exactas y Naturales, Universidad Estatal a Distancia, Montes de Oca, San José, Costa Rica. \\ 3. Escuela de Biología, Museo de Zoología, Universidad de Costa Rica, Montes de Oca, San José, Costa Rica; \\ darha.s.u@gmail.com \\ 4. Centro de Investigación en Ciencias del Mar y Limnología, Universidad de Costa Rica, Montes de Oca, San José, \\ Costa Rica; monika.springer@ucr.ac.cr
}

Recibido 12-II-2020. Corregido 07-VII-2020. Aceptado 31-VIII-2020.

\begin{abstract}
First record of the genus Interocoris (Heteroptera: Naucoridae) from Central America and observations on Interocoris mexicanus. Introduction: The family Naucoridae, of worldwide distribution, is divided into five subfamilies, with four of them and six genera reported to date in Central America. The genus Interocoris (Laccocorinae) is monotypic and was only recorded from Mexico. Objective: To report the first confirmed record of Interocoris mexicanus from Costa Rica and Central America, and to provide complementary descriptions of adults and nymphal instar V and associated microhabitat. Methods: a comparison of the external morphology (measurements and proportions) was made of specimens collected from two localities in Costa Rica with the type material of I. mexicanus from Mexico. Results: a positive identification of the species was obtained from the morphological comparison, which is why it is reported for the first time from Costa Rica and Central America. The specimens collected generally coincided with the measurements and relationships of the type material of I. mexicanus, with slight differences, some of them even reported for the specimens of the type series in the original description. Complementary descriptions of adults are presented and the morphology of nymph V is described for the first time. Conclusions: with this new record, the range of the genus Interocoris as well as the species I. mexicanus is expanded significantly from Mexico to Costa Rica. In addition, the reported number of genera of Naucoridae is increased to six in Costa Rica and to seven in the Central American region.
\end{abstract}

Key words: Laccocorinae; diversity; range expansion; Neotropics; aquatic insects.

Herrera, F., Solano-Ulate, D., \& Springer, M. (2020). Primer registro del género Interocoris (Heteroptera: Naucoridae) en Centroamérica y observaciones sobre Interocoris mexicanus. Revista de Biología Tropical, 68(Supl. 2), S150-S158.

La familia Naucoridae, de distribución mundial y con alrededor de 400 especies descritas, se divide en cinco subfamilias, de las cuales cuatro se encuentran representadas en Centroamérica por seis géneros Ambrysus Stål, Cryphocricos Signoret, Limnocoris Stål, Pelocoris Stål, Ctenipocoris Montandon y Cataractocoris Usinger (Herrera, 2013a; Herrera, 2013b). Este último género no ha sido registrado en Costa Rica. El género Interocoris La Rivers (1974) pertenece a la subfamilia Laccocorinae, con diez géneros alrededor del mundo, de los cuales tres tienen representantes en el continente americano (Sites et al., 2011). Decarloa La Rivers se encuentra en Haití y República Dominicana (Ludwick \& Sites, 2015), Ctenipocoris en Sudamérica y Costa Rica (Herrera, 2013b) y el monotípico 
Interocoris ha sido registrado únicamente en México (Sites \& Camacho, 2014). Interocoris mexicanus Usinger (1935) ha sido registrado para el Estado de México, Chiapas, Guerrero, Jalisco, Nayarit, Oaxaca y Sonora (Usinger, 1935; Polhemus \& Polhemus, 2008; ReynosoVelasco \& Sites, 2019). En este trabajo, se registra por primera vez I. mexicanus en Costa Rica siendo éste el primer registro de la especie en Centroamérica. Además, se proporcionan descripciones e ilustraciones complementarias de la especie.

\section{MATERIALES Y MÉTODOS}

Los ejemplares se recolectaron directamente del sustrato utilizando pinzas. Se les midió la longitud y ancho del cuerpo, así como de sus estructuras principales con la ayuda de un lente micrométrico. Las mediciones de longitud fueron tomadas a lo largo de la línea media y los anchos perpendiculares a éstas. $\mathrm{La}$ numeración de los segmentos abdominales se da en números romanos. La identificación de los especímenes se realizó con la información propuesta por Usinger (1935) en la descripción original de la especie. Se consultó el material tipo depositado en la Academia de Ciencias de California (CAS), San Francisco, California, Estados Unidos. No se realizó disección del material tipo en cuanto a genitales y las mediciones se basan en las reportadas en la descripción original de la especie. Se siguió la terminología utilizada por De Carlo (1965) para nombrar los segmentos genitales, así como el contenido de la cápsula genital. Se utilizó el software Macromedia Flash Professional 8.0. para realizar las ilustraciones. No se brindan mediciones para una de las hembras por encontrarse en mal estado y con faltantes de partes corporales. Todos los ejemplares se preservan en etanol al 70\% y se encuentran depositados en la Colección de Entomología Acuática del Museo de Zoología de la Universidad de Costa Rica (MZUCR).

Material examinado: COSTA RICA. GUANACASTE, Bagaces, Catarata
Llanos de Cortés, Río Potrero (10³1'28.84”N; $\left.85^{\circ} 17^{\prime} 54.70^{\prime \prime} \mathrm{W}\right), 85$ m.s.n.m. 23.I.2019. Col. D. Solano-Ulate, $1 \delta^{\lambda}, 3$, 1 ninfa V; Santa Cruz, Parque Nacional Diriá, Catarata El Brasil, Río Brasil (1009'58.14”N; 85³5'38.51'”W), 160 m.s.n.m. 09.XII.2013. Cols. F. Vásquez \& J. Corrales, $1 \hat{O}, 1$ ninfa III.

Material adicional examinado: MÉXICO. ESTADO DE MÉXICO, Distrito de Temescaltepec, Río de Temascaltepec, cerca del pueblo El Tule, 1524 m.s.n.m. 2.VI.1933. Cols. H. E. Hinton \& R. L. Usinger, 10 (HOLOTIPO, CAS); 19 (ALOTIPO, CAS); $1{ }^{\Uparrow}$ (PARATIPO, CAS). SINALOA, $14.5 \mathrm{Km}$ al Este de Chupaderos, 19.V.1962. Col. F. D. Parker \& L. A. Stange, $2 \widehat{\jmath}$ (CAS). Sin datos de

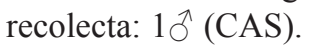

\section{RESULTADOS}

Mediciones detalladas de los ejemplares se brindan en el Tabla 1. Los ejemplares son coincidentes con la descripción realizada por Usinger (1935) en cuanto al aspecto general y características morfológicas. Se describen características complementarias para cada sexo.

Machos: longitud promedio de $7.82 \mathrm{~mm}$ y $4.57 \mathrm{~mm}$ de ancho (abdomen). Se observan cuatro depresiones equidistantes a lo largo del margen interno de cada ojo; las tres primeras son circulares; la cuarta depresión es de forma sub-triangular o con forma de "V" invertida (con el ápice dirigido hacia el espacio interocular) cerca de la unión del margen interno con el posterior; se evidencian mechones de sedas saliendo de cada depresión, tal y como se observa también en las hembras (Fig. 1). Relaciones corporales obtenidas: ancho/longitud del labro es 2.2; longitud del pronoto/longitud de cabeza es 1.8; ancho pronoto/longitud del pronoto es 2.5; ancho/longitud de escutelo es 1.95; longitud escutelo/longitud pronoto es 0.9. Los ángulos posterolaterales de los segmentos abdominales (II-VI) forman puntas redondeadas. La lengüeta o cono anal, de la cápsula genital del macho, es triangular con dos 
TABLA 1

Mediciones descriptivas de individuos de Interocoris mexicanus de Cascadas Llanos de Cortés y El Brasil, Guanacaste, Costa Rica. Mediciones dadas en milímetros y desviación estándar entre paréntesis.

Mediciones de cuerpo, cabeza y notales, tomadas en la línea media

TABLE 1

Descriptive measurements of individuals of Interocoris mexicanus from Llanos de Cortés and El Brasil waterfalls, Guanacaste, Costa Rica. Units given in millimeters and standard deviation inside brackets. Body, head and notal measurements taken at midline

\begin{tabular}{|c|c|c|}
\hline Caracteres & Macho $(n=2)$ & Hembra $(n=2)$ \\
\hline Cuerpo (longitud total) & $7.82( \pm 0.47)$ & $7.66( \pm 0.21)$ \\
\hline Cuerpo (ancho en abdomen) & $4.57( \pm 0.13)$ & $4.53( \pm 0.00)$ \\
\hline Cabeza (longitud) & $0.90( \pm 0.09)$ & $0.84( \pm 0.02)$ \\
\hline Cabeza incl. ojos (ancho) & $3.08( \pm 0.04)$ & $3.10( \pm 0.02)$ \\
\hline Ojo (longitud) & $0.70( \pm 0.02)$ & $0.70( \pm 0.02)$ \\
\hline Ojo (ancho) & $0.82( \pm 0.04)$ & $0.83( \pm 0.02)$ \\
\hline Distancia interocular anterior & $1.40( \pm 0.00)$ & $1.38( \pm 0.01)$ \\
\hline Distancia interocular posterior & $1.52( \pm 0.02)$ & $1.54( \pm 0.00)$ \\
\hline Labro (longitud) & $0.32( \pm 0.00)$ & $0.33( \pm 0.01)$ \\
\hline Labro (ancho) & $0.88( \pm 0.02)$ & $0.88( \pm 0.02)$ \\
\hline Pronoto (longitud) & $1.62( \pm 0.02)$ & $1.60( \pm 0.03)$ \\
\hline Pronoto (ancho) & $4.25( \pm 0.10)$ & $4.25( \pm 0.00)$ \\
\hline Escutelo (longitud) & $1.47( \pm 0.13)$ & $1.30( \pm 0.15)$ \\
\hline Escutelo (ancho) & $2.85( \pm 0.25)$ & $2.55( \pm 0.00)$ \\
\hline Sutura claval (longitud) & $0.87( \pm 0.08)$ & $0.90( \pm 0.00)$ \\
\hline Hemiélitro (longitud) & $3.12( \pm 0.08)$ & $3.08( \pm 0.04)$ \\
\hline Hemiélitro (ancho en membrana) & $1.34( \pm 0.02)$ & $1.32( \pm 0.00)$ \\
\hline Profémur (longitud) & $1.55( \pm 0.06)$ & $1.52( \pm 0.02)$ \\
\hline Profémur (ancho) & $0.84( \pm 0.04)$ & $0.87( \pm 0.03)$ \\
\hline Mesofémur (longitud) & $1.71( \pm 0.00)$ & $1.71( \pm 0.00)$ \\
\hline Mesofémur (ancho) & $0.61( \pm 0.01)$ & $0.61( \pm 0.00)$ \\
\hline Metafémur (longitud) & $2.12( \pm 0.02)$ & $2.09( \pm 0.05)$ \\
\hline Metafémur (ancho) & $0.62( \pm 0.00)$ & $0.61( \pm 0.01)$ \\
\hline Cantidad uñas en protarso & 2 & 2 \\
\hline Cantidad de segmentos tarsales en propata & 2 & 1 \\
\hline
\end{tabular}

engrosamientos cuticulares que penetran hasta casi el primer tercio basal (Fig. 2A). Edeago ligeramente cuadrangular en su parte distal con el ápice suavemente redondeado, más angosto en su base, con una proyección subtrapezoidal en su costado derecho, así como otra proyección triangular cercana al ápice y a la posición central; los harpagones o parámeros son simétricos, sin tocarse o cruzarse y con la base en forma de gancho; el borde superior del esternito basal del pigóforo es ligeramente trisinuado (Fig. 2B). Se observan perforaciones finas en los harpagones como en el esternito basal, especialmente abundantes en las partes oscuras. La carena o quilla mesosternal, en vista lateral, comienza una curva cóncava que en su extremo posterior forma un diente anterior conspicuo de punta redondeada; la parte central de la quilla se eleva de manera convexa superando, en su primer tercio, la altura del diente anterior; el último tercio presenta la mayor altura y luego decrece abruptamente de manera redondeada; diente posterior formado un ángulo de $90^{\circ}$ (Fig. 2C). Se observan sedas, de longitud igual a la misma quilla, saliendo radialmente en todas las direcciones. 


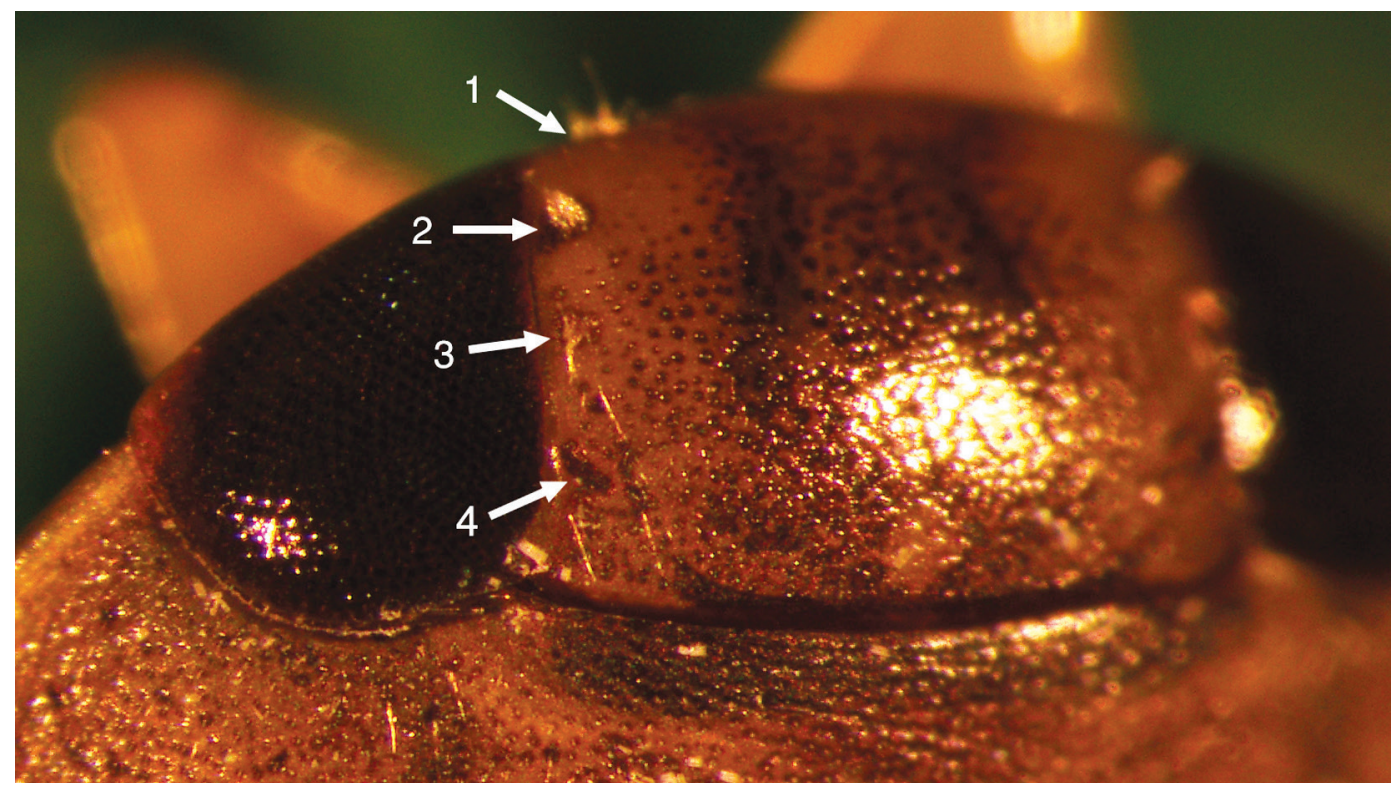

Fig. 1. Vista dorsal del detalle de las cuatro depresiones a lo largo del margen interno del ojo (indicadas con números), del espécimen hembra de Interocoris mexicanus recolectada en Cascada Llanos de Cortés, Guanacaste, Costa Rica. Longitud de la cabeza en línea media: $0.86 \mathrm{~mm}$.

Hembras: longitud promedio de $7.66 \mathrm{~mm}$ y $4.53 \mathrm{~mm}$ de ancho (abdomen). En una de las hembras, las depresiones a lo largo del margen interno de los ojos, son semejantes a las descritas para los machos anteriormente; la otra hembra presenta cinco depresiones, misma situación que en la ninfa $\mathrm{V}$ descrita más abajo, excepto que, en esta hembra, la quinta depresión está reducida a un cuadrado en el lado izquierdo y muy poco evidente en el lado derecho. Relaciones corporales y ángulos posterolaterales del abdomen (II-VI) muy similares a las obtenidas para los machos. Placa subgenital (esternito VII) cubierta de sedas; con una anchura máxima en la base de 1.19 $\mathrm{mm}$; una longitud en su línea media de 1.01 $\mathrm{mm}$; los tercios basal y apical son ligeramente convexos, mientras que el tercio medial es ligeramente cóncavo; el margen posterior es de recto a ligeramente sinuado (Fig. 2D). La quilla mesosternal es similar a la descrita para el macho. La hembra incompleta presentaba dentro de su abdomen un huevo, de su especie, de color amarillento claro, muy ovalado, con una longitud de $1.44 \mathrm{~mm}$ y un ancho de $0.69 \mathrm{~mm}$.

Alas: Todos los individuos recolectados son macrópteros. Ala posterior con longitud máxima de $4.97 \mathrm{~mm}$; anchura máxima de 3.54 $\mathrm{mm}$. La vena Subcostal (Sc), la Radial $\left(\mathrm{R}_{1}\right)$ y la medial $\left(\mathrm{M}_{1+2}\right)$, se unen formando un lazo. Además, la cubital $(\mathrm{Cu})$ y la primera anal $\left(\mathrm{A}_{1}\right)$ están completas y bien formadas, y entre ellas se observan dos venas auxiliares formando una celda bien delimitada (Fig. 3).

Ninfa V: Mediciones detalladas del espécimen en el Tabla 2. Longitud de $6.15 \mathrm{~mm}$; ancho $4.26 \mathrm{~mm}$ (embolio); cuerpo oblongo y aplanado dorsoventralmente excepto cerca del metanoto en donde se vuelve ligeramente convexo. Superficie dorsal con coloración amarillenta tornándose más blancuzca hacia los bordes; presenta manchas café-negruzcas y toda su superficie perforaciones pardo oscuras; escasa pubescencia dorsal; un mechón 
A

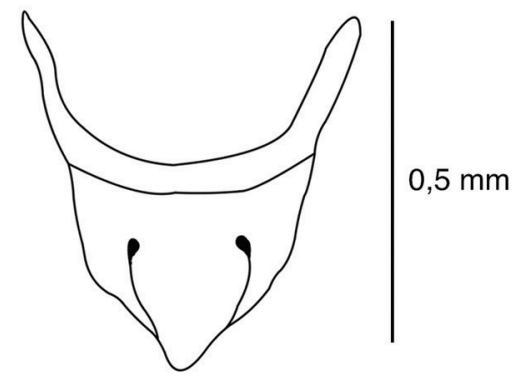

C

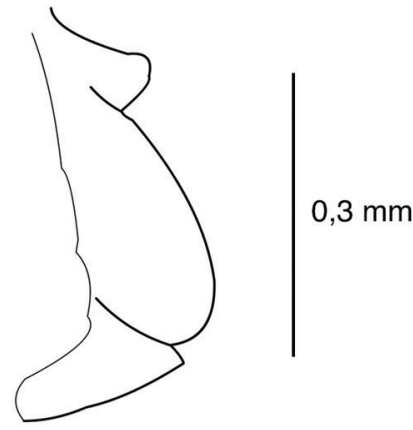

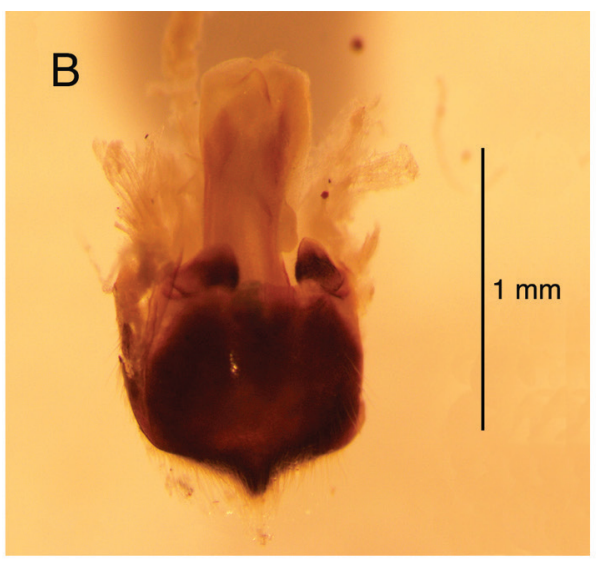

D

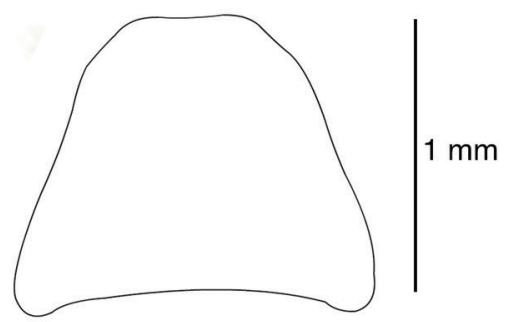

Fig. 2. A. Lengüeta o cono anal en vista dorsal de la cápsula genital y B. Cápsula genital del macho de Interocoris mexicanus, recolectado en Cascada Llanos de Cortés, Guanacaste, Costa Rica. C. Quilla mesosternal en vista lateral, y D. Placa subgenital (no aplanada) de la hembra de Interocoris mexicanus recolectada en Cascada Llanos de Cortés, Guanacaste, Costa Rica.

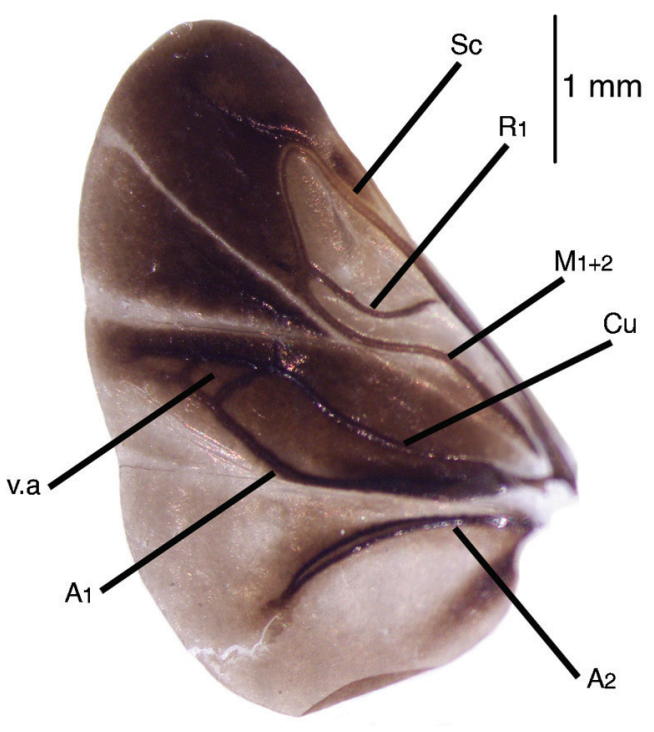

de sedas saliendo de cada uno de los ángulos posterolaterales del abdomen (II-VI). Cabeza con cinco depresiones equidistantes a lo largo del margen interno de los ojos, de los cuales salen mechones de sedas; las primeras cuatro depresiones son similares a las descritas para el macho; la quinta depresión tiene forma de línea corta y está al lado de la cuarta depresión, pero no a lo largo del margen interior del ojo, sino más bien hacia adentro del espacio interocular (Fig. 4); borde interno de los ojos

Fig. 3. Ala posterior de Interocoris mexicanus, de un espécimen hembra recolectado en Cascada Llanos de Cortés, Guanacaste, Costa Rica. Siendo las venas, Sc: Subcostal, $\mathrm{R}_{1}$ : Radial, $\mathrm{M}_{1+2}$ : Medial, Cu: Cubital, v.a: Celda auxiliar, $\mathrm{A}_{1}$ : primera Anal; $\mathrm{A}_{2}$ : segunda Anal. 
TABLA 2

Mediciones de un ejemplar ninfa del estadio $\mathrm{V}$ de Interocoris mexicanus de Cascadas Llanos de Cortés, Guanacaste, Costa Rica. Unidades dadas en milímetros. Mediciones de cuerpo, cabeza y notales, tomadas en la línea media

TABLE 2

Descriptive measurements of instar V of Interocoris mexicanus from Llanos de Cortés and El Brasil waterfalls, Guanacaste, Costa Rica. Units given in millimeters. Body, head and notal measurements taken at midline

\begin{tabular}{lc}
\multicolumn{1}{c}{ Caracteres } & Ninfa V (n=1) \\
Cuerpo (longitud total) & 6.15 \\
Cuerpo (ancho embolio) & 4.26 \\
Cabeza (longitud) & 0.94 \\
Cabeza incl. ojos (ancho) & 2.68 \\
Ojo (longitud) & 0.79 \\
Ojo (ancho) & 0.79 \\
Distancia interocular & 1.35 \\
Pronoto (longitud) & 0.95 \\
Pronoto (ancho) & 3.73 \\
Mesonoto (longitud) & 0.95 \\
Metanoto (longitud) & 0.79 \\
Labro (longitud) & 0.23 \\
Labro (ancho) & 0.66 \\
Profémur (longitud) & 1.16 \\
Protibia (longitud) & 0.70 \\
Protarso (longitud) & 0.23 \\
Mesofémur (longitud) & 1.33 \\
Mesotibia (longitud) & 1.03 \\
Mesotarso (longitud) & 0.5 \\
Metafémur (longitud) & 1.66 \\
Metatibia (longitud) & 1.83 \\
Metatarso (longitud) & 0.83 \\
Cantidad uñas en protarso & 2 \\
\hline
\end{tabular}

subparalelos. Se concluye que se trata del estadio ninfal $\mathrm{V}$ debido a que los parches alares alcanzan hasta el borde posterior del metanoto. Relaciones corporales obtenidas: longitud del pronoto/longitud del mesonoto es 1.0; longitud del mesonoto/longitud del metanoto es 1.2. Ventralmente es de color amarillento excepto en el abdomen el cual es pardo oscuro. Labro redondeado en su borde distal. Relación longitud de profémur/longitud de protibia es 1.6. Segmento tarsal de la propata unisegmentado con dos uñas. Quilla mesoesternal con forma

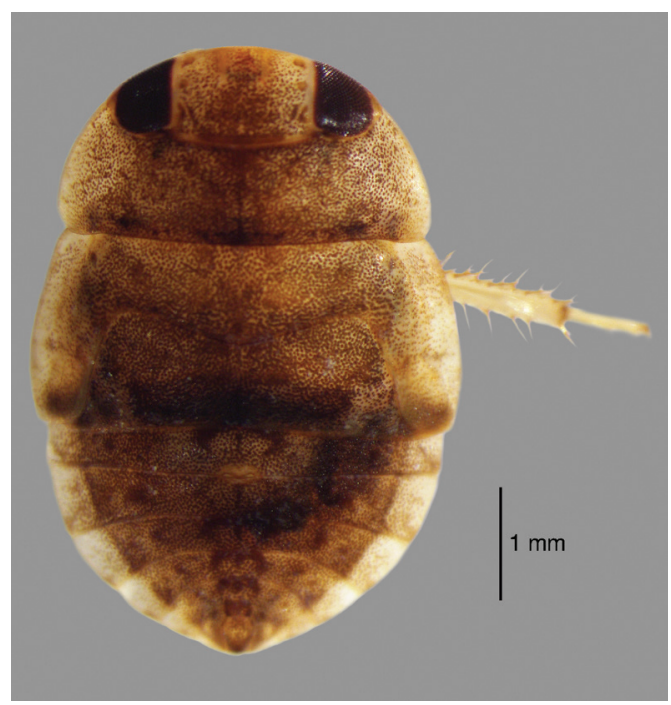

Fig. 4. Ninfa V de Interocoris mexicanus, recolectado en Cascada Llanos de Cortés, Guanacaste, Costa Rica.

de pirámide, pero su cara posterior finaliza de forma más abrupta que las demás caras; ápice de la quilla con sedas con una longitud cercana a la mitad de la altura de la misma. Abdomen con pubescencia muy corta; bordes externos del abdomen sin pubescencia lo cual hace que tome una coloración blancuzca.

\section{DISCUSIÓN}

Los especímenes adultos encontrados en las cataratas de Llanos de Cortés y El Brasil coinciden con cinco de las ocho relaciones corporales de los ejemplares tipo de la especie descrita por Usinger (1935). Entre otros rasgos dorsales coincidentes están: coloración dorsal; patrón de perforación del cuerpo; cuatro depresiones equidistantes en la cabeza de las cuales salen mechones de sedas en tres de los ejemplares; forma de los márgenes laterales, ángulos antero- y posterolaterales del pronoto, así como la mancha circular más clara en su línea media; forma de márgenes y elevación del escutelo; forma de hemiélitros. Ventralmente coincidieron los siguientes rasgos: coloración y pubescencia; forma y características de las patas; distribución de las espinas y sedas; segmentos tarsales de la propata, dos en el macho 


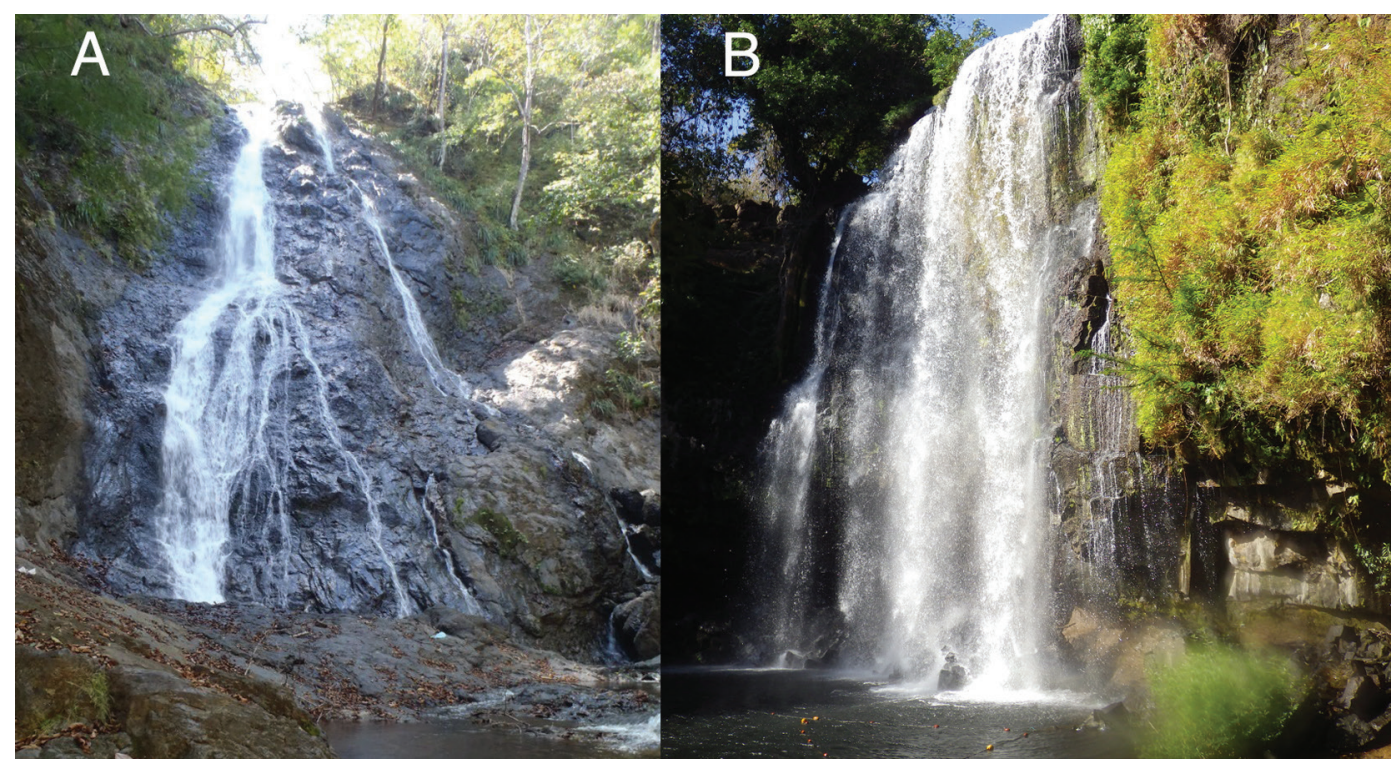

Fig. 5. Sitios de recolecta de Interocoris mexicanus, Guanacaste, Costa Rica. A) Cascada El Brasil, B) Cascada Llanos de Cortés. Fotos: F. Vásquez \& M. Blanco.

y uno en la hembra; tarsos de la pata delantera con dos uñas.

No obstante, se observan algunas diferencias en el tamaño, siendo, en promedio, ligeramente más pequeños los organismos recolectados en este estudio. Además, no se observó el pequeño proceso externo del ojo, y los ángulos posterolaterales de los segmentos abdominales II-VI formaron puntas redondeadas y no ángulos rectos conspicuos. Las relaciones no coincidentes con la descripción original de la especie son las relaciones corporales: ancho/longitud de cabeza; ancho/ longitud de espacio interocular; longitud de escutelo/longitud de sutura claval.

Por otro lado, la posición general de las venas de las alas coincide con la descrita por Hoke (1926) para la familia. En I. mexicanus la $\mathrm{R}_{1}$ se separa de la $\mathrm{Sc}$ formando la celda radial; la $\mathrm{Cu}$ y la $\mathrm{A}_{1}$ están completas y bien formadas, y entre ellas se observa una celda auxiliar bien definida formada por dos venas auxiliares. La venación de esta ala coincide en gran medida con el ala de Namtokocoris siamensis Sites, perteneciente a la subfamilia Laccocorinae, excepto que en éste último no se forma una vena auxiliar (Sites \& Vitheepradit, 2007). Además, con respecto a la forma de la quilla mesosternal, ésta podría tener una especial importancia para la identificación de futuras especies como sucede en el género americano Limnocoris (Nieser \& López Ruf, 2001; Rodrigues \& Sites, 2019).

Los especímenes de I. mexicanus se recolectaron en paredes de cascadas (Fig. 5), tanto en zonas de salpique como de flujo laminar. Así mismo, los organismos de I. mexicanus se encontraron inmersos dentro del limo que se encontraba cubriendo la roca, así como plantas acuáticas, algas y musgos. Esto coincide con la información sobre el tipo de microhábitat reportado por Polhemus y Polhemus (2008), pero difiere del reportado por Usinger (1935) para la serie tipo, los cuales fueron encontrados en limo a lo largo de los márgenes del río. Otros naucóridos americanos del género Cataractocoris Usinger, fueron recolectados en condiciones muy similares (Usinger, 1941; Sites, 2004; Herrera; 2016 \& Sites et al. 2016), lo que sugiere que estos géneros pueden estar asociados principalmente a estos ambientes poco estudiados. 
Conclusión: Aunque no se realizaron comparaciones de las estructuras genitales del holotipo macho de I. mexicanus con las de los ejemplares recolectados en Costa Rica, la correspondencia de las medidas, proporciones y morfología externa en general nos permite realizar una identificación positiva de nuestros ejemplares. No obstante, consideramos importante que en el futuro próximo se realice una descripción detallada de las estructuras de la terminalia y genitalia de hembras y machos de esta especie, lo cual proporcionará caracteres más estables para la correcta identificación de la especie.

De esta forma, con este registro se aumentan a seis los géneros de Naucoridae registrados para Costa Rica y se actualiza la información presentada por Springer (2019). Del mismo modo, se aumenta a siete el número de géneros de Naucoridae reportados para Centroamérica y se amplía el rango de distribución de Interocoris el cual va desde Sonora, al norte de México, hasta Guanacaste, en el norte de Costa Rica. Es de esperar que ejemplares de esta especie sean encontrados en los cinco países intermedios entre México y Costa Rica.

Finalmente, consideramos de suma importancia el estudio de los diferentes microhábitat presentes en las cascadas a lo largo del país para aumentar el conocimiento sobre los taxones que comparten esos microhábitat y descubrir las posibles relaciones ecológicas que se estén llevando a cabo entre ellos.

Declaración de ética: los autores declaran que están de acuerdo con esta publicación; que no existe conflicto de interés de ningún tipo; y que ha cumplido con todos los requisitos y procedimientos éticos y legales pertinentes. Todas las fuentes de financiamiento se detallan plena y claramente en la sección de agradecimientos. El respectivo documento legal firmado se encuentra en los archivos de la revista.

\section{AGRADECIMIENTOS}

Los autores agradecen a la Municipalidad de Bagaces y Rosbin Rojas por permitir el acceso al sitio de recolecta; al Sistema Nacional de Áreas de Conservación (SINAC), dependencia del Ministerio de Ambiente y Energía (MINAE) por el otorgamiento de los permisos de recolecta; a los revisores del manuscrito cuyas detalladas sugerencias ayudaron a mejorarlo en gran medida.

\section{RESUMEN}

Introducción: La familia Naucoridae, de distribución mundial, se encuentra dividida en cinco subfamilias, de las cuales cuatro de ellas y seis géneros han sido reportados hasta la fecha en Centroamérica. El género Interocoris (Laccocorinae) es monotípico y anteriormente sólo se había registrado en México. Objetivo: reportar el primer registro confirmado de I. mexicanus para Costa Rica y Centroamérica, así como proporcionar descripciones complementarias de los adultos, ninfa V y microhábitat asociado. Métodos: se realizó una comparación de la morfología externa (medidas y proporciones) de los ejemplares recolectados en dos localidades de Costa Rica con la información y el material tipo de I. mexicanus, recolectado en México. Resultados: A partir de la comparación morfológica se obtuvo una identificación positiva de la especie, por lo que se reporta por primera vez a I. mexicanus de Costa Rica y Centroamérica. Los especímenes recolectados coincidieron en general con las mediciones y relaciones del material tipo de $I$. mexicanus, con ligeras diferencias, algunas de ellas incluso reportadas para los ejemplares de la serie tipo en la descripción original. Se presentan descripciones complementarias de adultos y se describe por primera vez la morfología de la ninfa V. Conclusiones: con este nuevo registro se expande significativamente el rango del género Interocoris, así como de la especie I. mexicanus desde México a Costa Rica. Además, se aumentan a seis los géneros de Naucoridae en Costa Rica y a siete en Centroamérica.

Palabras clave: Laccocorinae; diversidad; expansión del rango; Neotrópico; insectos acuáticos.

\section{REFERENCIAS}

De Carlo, J. A. (1965). Segmentos genitales y contenido de la cápsula genital en machos de especies de los géneros Ambrysus, Pelocoris, Limnocoris, Heleocoris y Cryphocricos (Hemiptera - Naucoridae). Revista de la Sociedad Entomológica Argentina, 27(1-4), 101-106.

Herrera, F. (2013a). Estado del conocimiento y catálogo de la familia Naucoridae (Insecta: Heteroptera) en Centroamérica. Dugesiana, 20(2), 221-232.

Herrera, F. (2013b). First record of the genus Ctenipocoris (Heteroptera: Naucoridae) in Central America, with 
a preliminary key to the American species and descriptions of a new species. Zootaxa, 3731, 338-344.

Herrera, F. (2016). Distributional range expansion of Cataractocoris marginiventris Usinger (Hemiptera: Heteroptera: Naucoridae) and first record from El Salvador. Acta Zoológica Mexicana (Nueva Serie), 32(1), 111-112.

Hoke, S. (1926). Preliminary paper on the wing-venation of the Hemiptera (Heteroptera). Annals of the Entomological Society of America, 19, 13-34.

La Rivers, I. (1969). New naucorid taxa (Hemiptera). Biological Society of Nevada (Occasional Papers), 20, 1-12.

La Rivers, I. (1974). Catalogue of taxa described in the family Naucoridae (Hemiptera). Supplement No. 1: corrections, emendations and additions, with descriptions of new species. Occasional Papers of the Biological Society of Nevada, 38, 1-17.

Ludwick, D. C., \& Sites, R. W. (2015). Descriptions of the female and nymphal instars of Decarloa darlingtoni La Rivers (Hemiptera: Heteroptera: Naucoridae). Proceedings of the Entomological Society of Washington, 117(1), 14-21.

Montandon, A. L. (1897). Hemiptera cryptocerata. Fam. Naucoridae. - Sous-fam. Laccocorinae. Verhandlungen der Zoologisch-Botanischen Gesellschaft in Wien, 47: 435-454.

Nieser, N., \& López Ruf, M. (2001). A review of Limnocoris Stål (Heteroptera: Naucoridae) in Southern South America East of the Andes. Tijdschrift voor Entomologie, 144: 261-328,

Polhemus, J. T., \& Polhemus, D. A. (2008). Intraspecific morphological polymorphism in Naucoridae (Hemiptera: Heteroptera) with notes on nomenclature and synonymy. Acta Entomologica Musei Nationalis Pragae, 48(2), 289-298.

Reynoso-Velasco, D., \& Sites R. W. (2019). Taxonomic overview of the family Naucoridae (Heteroptera: Nepomorpha) in Mexico. Dugesiana, 26(1), 3-12.

Rodrigues, H. D. D., \& Sites, R. W. (2019). Revision of Limnocoris (Heteroptera: Nepomorpha: Naucoridae) of North America. Zootaxa, 4629(4), 451-497.

Signoret, M. V. (1850). Description d'un genre nouveau de l'ordre des Hémiptères hétéroptères, et de la section des Hydrocoryses. En M. F. E. Guérin Meneville \& M. A. Focillon (Eds.), Revue et Magasin de Zoologie Pure et Appliqué. 2 ${ }^{\mathrm{e} S e ́ r i e . ~(T . ~ I I, ~ p p . ~ 289-291) . ~ P a r i s, ~}$ Francia: Bureau de la Revue et Magasin de Zoologie.

Sites, R. W. (2004). Distributional records of Cataractocoris (Heteroptera: Naucoridae) in Mesoamerica. Entomological News, 115(3), 173-174.

Sites, R.W., \& Camacho, J. (2014). Neotropical genera of Naucoridae (Hemiptera: Heteroptera: Nepomorpha): New species of Placomerus and Procryphocricos from Guyana and Venezuela. Zootaxa, 3753(5), 469-482.

Sites, R. W., Reynoso-Velasco, D. \& Novelo-Gutiérrez, R. (2013). Revision of the Mesoamerican Genus Cataractocoris Usinger (Hemiptera: Heteroptera: Nepomorpha: Naucoridae). Zootaxa, 3682(3), 475-484.

Sites, R.W., \& Vitheepradit, A. (2007). Namtokocoris Sites, a new genus of Naucoridae (Hemiptera: Heteroptera) in waterfalls of Indochina, with descriptions of six new species. Zootaxa, 1588, 1-29.

Sites, R.W., Zettel, H., \& Arunachalam, M. (2011). Waterfall-inhabiting Naucoridae (Hemiptera: Heteroptera) of southern India and Sri Lanka: Pogonocaudina Sites and Zettel, n. gen., and a review of Diaphorocoris with descriptions of two new species. Zootaxa, 2760, 1-17.

Springer, M. (2019). La colección de Entomología Acuática del Museo de Zoología, Universidad de Costa Rica: lista actualizada de géneros, importancia y retos futuros. Revista de Biología Tropical, 67(2), 200-211.

Stål, C. (1862). Hemiptera mexicana enumeravit speciesque novas descriptsit. Stettiner Entomologische Zeitung, 23, 437-462.

Stål, C. (1876). Enumeratio Hemipterorum. Bidragtill en förteckning öfver alla hittills kända Hemiptera, jemte systematiska meddelanden. Köngliga Svenska Vetenskaps-Akademiens Handlingar, 14(4), 141-147.

Usinger, R. L. (1935). A second American species of the Naucorid subfamily Laccocorinae (Hemiptera). Revista de Entomologia, 5(2), 133-136.

Usinger, R. L. (1941). Key to the subfamilies of Naucoridae with a generic synopsis of the new subfamily Ambrysinae (Hemiptera). Annals of the Entomological Society of America, 34(1), 5-16. 